\title{
The Effect of Military Load Carriage on Ground Reaction Forces
}

Technical Note

\author{
Stewart A Birrell ${ }^{1}$ \\ Robin H Hooper ${ }^{1}$ \\ Roger A Haslam ${ }^{1}$
}

${ }^{1}$ Department of Human Sciences, Loughborough University, Leicestershire, LE11 3TU, UK

Keywords: Load Carriage, Ground Reaction Force, Military, Gait, Rifle Carriage 


\begin{abstract}
Load carriage is an inevitable part of military life both during training and operations. Loads carried are frequently as high as $60 \%$ bodyweight, and this increases injury risk. In the military, load is carried in a backpack (also referred to as a Bergen) and webbing, these combined form a load carriage system (LCS). A substantial body of literature exists recording the physiological effects of load carriage; less is available regarding the biomechanics. Previous biomechanical studies have generally been restricted to loads of 20 and $40 \%$ of bodyweight, usually carried in the backpack alone. The effect of rifle carriage on gait has also received little or no attention in the published literature. This is despite military personnel almost always carrying a rifle during load carriage. In this study 15 male participants completed 8 conditions: military boot, rifle, webbing 8 and $16 \mathrm{~kg}$, backpack $16 \mathrm{~kg}$, and LCS 24, 32 and $40 \mathrm{~kg}$. Results showed that load added in $8 \mathrm{~kg}$ increments elicited a proportional increase in vertical and anteroposterior ground reaction force (GRF) parameters. Rifle carriage significantly increased the impact peak and mediolateral impulse compared to the boot condition. These effects may be the result of changes to the vertical and horizontal position of the body's centre of mass, caused by the restriction of natural arm swing patterns. Increased GRFs, particularly in the vertical axis, have been positively linked to overuse injuries. Therefore, the biomechanical analysis of load carriage is important in aiding our understanding of injuries associated with military load carriage.
\end{abstract}

\title{
Introduction
}

Military mission requirements often depend on personal mobility. In these situations personnel carry their own equipment, usually in a backpack (Bergen) and webbing, so forming a load carriage system (LCS). A rifle is also carried on most occasions when marching. The study of ground reaction forces (GRF) during load carriage can provide relevant information about the mechanisms of gait, and provide a 
measure of the impact forces acting on the foot. It is therefore essential in the understanding and prevention of lower extremity injuries [1].

Research investigating the effect of load carriage on GRFs and gait is not widely represented within the literature $[1,2,3,4,5,6]$. However, conclusions drawn confirm, that as would be expected, both vertical and anteroposterior GRFs produced during gait increase when load is applied to the body. However, the proportionality or rate of this increase has been debated within the literature. The majority of research suggests that the increase in vertical and anteroposterior GRFs is directly proportional to the applied load $[2,3,4]$. These studies suggest that $1 \mathrm{~kg}$ of added load equates to approximately a $10 \mathrm{~N}$ increase in force. Other studies suggest that protective mechanisms, such as an increase in double support or decreased walking speed, are activated when carrying heavy loads in an effort to reduce stresses placed on the lower extremities $[1,5]$. Finally, changes to the GRF parameters of the mediolateral axis have been found to be insignificant $[1,4,6]$.

The primary aim of this research was to examine the effect of progressive $8 \mathrm{~kg}$ increments in carried load on GRF parameters. This would help establish base-line GRF data for load carried using the U.K '90 Pattern LCS, and investigate heavy military load carriage. The study design allowed other factors to be investigated including the effect of changing the load distribution, and also the potential effects of rifle carriage on GRF parameters.

\section{Technical Description}

Fifteen male participants volunteered for the study (mass $83.2 \mathrm{~kg} \pm 10.0$ S.D., height $178.8 \mathrm{~cm} \pm 5.4$, age 27.8 years \pm 7.0 ). In order to comply with the granted ethical approval and for the \% bodyweight carried to be deemed acceptable, each participant had to weigh over $70 \mathrm{~kg}$. All participants also had previous experience of carrying backpacks, and were rear-foot strikers. A verbal and written explanation of the study was given, after which a health screen questionnaire was completed. Informed consent was obtained from all participants before commencing the trial. 
A Kistler ${ }^{\mathrm{TM}}$ force plate (Type 9286A, dimensions $60 \times 30 \times 5 \mathrm{~cm}$ ) was used in conjunction with a Coda ${ }^{\mathrm{TM}}$ Mpx30 Motion Analysis System to obtain GRF data. Eight channels of kinetic data were sampled by the force plate at $400 \mathrm{~Hz}$. This raw data were then processed via $\mathrm{A} / \mathrm{D}$ converters situated in the Coda Mpx30 and outputted into CODAmotion v6.64 software. The data were then exported to Microsoft ${ }^{\mathrm{TM}}$ Excel for analysis. The force plate was embedded in an $8.4 \mathrm{~m}$ walkway. This gave adequate distance before and after the force plate to achieve a natural gait pattern. To measure the walking speed of the participants three pairs of infra-red photoelectric cells (Brower $^{\mathrm{TM}}$ SpeedTrap II) were used placed $1.5 \mathrm{~m}$ apart from each other. One set recorded speed on approach to the force plate and the other after the force plate. Both speeds had to be within the desired range thus limiting the potential for acceleration or deceleration that would affect the GRFs produced.

The load was carried using a standard issue UK military '90 Pattern Short Back Bergen and PLCE (Personal Load Carrying Equipment) waist webbing, which, when worn together form a LCS. A replica SA80 assault rifle, weighing $2.1 \mathrm{~kg}$, was also carried in certain conditions. Participants also wore standard issue military leather boots and woollen socks throughout the duration of the study.

Each participant completed all 8 conditions (table 1), with 10 successful trials in each condition. The force data were sampled at $400 \mathrm{~Hz}$ and the target speed throughout was $1.5 \mathrm{~m} . \mathrm{s}-1( \pm 5 \%)$. A trial was deemed successful if the speed was attained, the participant's dominant foot struck cleanly on the force plate and if a natural gait pattern was maintained. To ensure participants had familiarised themselves with the load and walking speed an unlimited number of practice walks were allowed. The order the participants completed the conditions were randomised.

Insert Table 1 Here

The participant's kinetic data were normalised and expressed as Newton's per unit body mass (N.BM-1). Data from the boot condition were normalised to bodyweight (including clothes and boots), the other conditions to system weight (this is the weight of 
the rifle added to that of the participant). All data are expressed as N.BM-1 but as explained above this may either be the weight of the participant alone, or with the rifle.

The primary aim of the study was to examine the effects that small, incremental load increases of $8 \mathrm{~kg}$ have on selected GRF parameters. For this reason the boot and backpack condition were excluded from this particular section of the analysis (table 1). This is because the rifle condition was considered a more suitable control to the boot condition, as a rifle would be carried during each loading conditions. The backpack condition was also excluded, thus eliminating the issue of having two conditions where the carried load totalled $16 \mathrm{~kg}$. To assess the effects of carried load on GRF parameters a one-way MANOVA was undertaken. To determine significance between the conditions a Bonferroni corrected pairwise comparisons were also conducted. A Paired Student t-test was conducted to assess significance with rifle carriage and changing load distribution. For these comparisons the boot and backpack condition were re-introduced. Significance was accepted at the level of $\mathrm{p} \leq 0.05$ and all statistical testing was conducted using SPSS v12.0.

\section{Discussion}

\section{Effect of Load}

Increasing carried load has a significant overall effect on all the GRF parameters measured (table 2). In addition, the pairwise comparisons revealed that all parameters, with the exception of mediolateral impulse, significantly increased with incremental increases of $8 \mathrm{~kg}$. An increase in load has been shown to increase GRF consistently within the literature $[1,2,3,4,5,6]$. An increase in stance time was also observed, which has been observed within the literature [2,5]. Numerous studies $[1,4,6]$ have found changes to the mediolateral GRF parameters to be insignificant, and others did not even report the data. Results from this current study go against this idea as a significant increase in total mediolateral impulse was observed with load. The increase in mediolateral impulse observed here may be linked to a decrease in stability. This may be caused by the continual shift (in both the vertical and horizontal direction) of the body's 
centre of mass (CoM) further away from its neutral position when load is added. Research has shown that the less the CoM is displaced the greater the static stability of an individual when carrying load [7].

Insert Table 2 Here

As highlighted previously, the literature on the proportionality of the increase in GRF parameters with applied load is contradictory. Results from the present study support the hypothesis that increases in vertical and anteroposterior GRF with applied load represent a linear relationship when walking at $1.5 \mathrm{~m} . \mathrm{s}-1$, even when heavy loads of $40 \mathrm{~kg}$ are carried. This suggests that the increase in force is predominantly due to the static effect of the load rather than changes in acceleration of the system [2]. Figure 1 shows the linear increase in measured force against carried load.

Insert Figure 1 Here

High magnitudes or volumes of impact forces, like those experienced during load carriage or running, are a major risk factor for overuse injuries. In particular, stress fractures of the tibia and metatarsals and knee joint problems $[4,8,9]$. Military recruits can cover up to $11 \mathrm{~km}$ per day, which is equivalent to around 9,000 impacts [10]. For this reason it may be advantageous to have the ability to accurately predict the forces produced when heavy loads are carried over known distances. Establishing a doseresponse relationship for distance marched and load carried may be feasible. This would require the knowledge of the maximum stress or strain that can be placed on a bone or joint before stress fractures or joint degeneration are likely to occur. It would then be possible to calculate the number of impacts made and accurately estimate the peak force produced during these impacts. This may allow prediction of the number or severity of overuse injuries sustained during a forced march by military personnel. Other factors need to be taken into account such as prior exposure to marching and previous injury. However, training regimes could be adapted to reduce the risk of overuse injuries, and theoretical maximum distances marched while carrying specific loads could be drawn up. 
These distances or loads could then increase as training advances, as soldiers become more used to the physical activity and as increases in bone mineral density of the lower limb occur. Using linear regression analysis to calculate the increase in impact peak force with load gave this equation: Impact Peak $=(0.013 \times$ Load $)+1.223$. Load is measured in $\mathrm{kg}$ and the values for impact peak are expressed as N.BM-1. The proportion of variation which can be explained by this equation (R2) is 0.780 or $78 \%$.

\section{Rifle Carriage and Load Distribution}

As mentioned in the introduction the study design allowed the effect of rifle carriage and changes to load distribution, with their subsequent affect on GRF, to be analysed. The following section will highlight differences found with the current study, however, more detailed analysis is needed with future research. The effect of rifle carriage was examined by comparing the Boot and Rifle condition and, load distribution by comparing the Webbing 2 and Backpack conditions (table 1). Results in table 3 show that the rifle condition exhibited a greater impact peak, maximum propulsive force and mediolateral impulse, while decreasing the force minimum compared to the boot condition. The most likely mechanism behind these changes to GRF parameters with rifle carriage is either the restriction of natural arm swing patterns, or the load of the rifle being added to the anterior of the body.

Insert Table 3 Here

Carrying $16 \mathrm{~kg}$ in the webbing compared to the backpack lead to an increased impact peak in the vertical axis, and a reduction in stance time (table 3). Higher impact forces observed in the webbing condition, may be due to a larger component of the weight being over the striking foot at the time of initial contact. This is supported by other research that states when the CoM is shifted anteriorly, the force at heel strike is increased [11]. Stance time was also significantly longer when carrying the backpack compared to webbing. This occurrence has been observed before $[2,6]$, with a backpack showing a trend for longer stance times than with a double-pack (load distributed around the anterior and posterior of the trunk). Reason for this increase may be due to the extra 
time it takes to shift the CoM over the base of support, or an increased need for stability. Another factor may be as a result of increased dampening or flexion of the lower limb.

\section{Conclusions}

This study aimed to examine the effects of progressive increments in carried load on GRF parameters. Results from the study suggest that both vertical and anteroposterior GRF parameters increase proportionally when load is added in $8 \mathrm{~kg}$ increments to a UK standard issue '90 Pattern LCS. This increase is observed even when heavy loads of 40 $\mathrm{kg}$ are carried. Unlike many other studies significant increases in force generated in the mediolateral axis was also observed with increasing load. This may suggest a decrease in stability as greater loads are carried.

A new finding for this field of research is the effect of rifle carriage on GRFs. Rifle carriage caused an increase in the impact peak, maximum propulsive force and mediolateral impulse while decreasing the force minimum. These effects may be due to the forward shift in the CoM or more likely due to the restricted arm movements while carrying a rifle.

\section{Acknowledgements}

This work was sponsored by the UK Ministry of Defence under contract with Dstl.

\section{References}

[1] Harman E, Han K-H, Frykman P, Pandorf C. The effects of backpack weight on the biomechanics of load carriage. Technical Report No T00-17, U.S Army Research Institute of Environmental Medicine, MA. 2000: 1-71. 
[2] Kinoshita H. Effects of different loads and carrying systems on selected biomechanical parameters describing walking gait. Ergonomics 1985; 28: 1347-1362.

[3] Tilbury-Davis D.C, Hooper RH. The kinetic and kinematic effects of increasing load carriage upon the lower limb. Human Movement Science 1999; 18: 693-700.

[4] Polcyn A, Bensel C, Harman E, Obusek J, Pandorf C, Frykman P. Effects of weight carried by soldiers: Combined analysis of four studies on maximal performance, physiology, and biomechanics. Technical Report No TR-02/010 US Army Research Institute of Environmental Medicine, MA. 2002: 1-64.

[5] Weise-Bjornstal D, Dufek J. The effect of weightload and footwear on the kinetic and temporal factors in level grade backpacking. Journal of Human Movement Science 1991; 21: $167-181$.

[6] Lloyd R, Cooke CB. Kinetic changes associated with load carriage using two rucksack designs. Ergonomics 2000; 43: 1331-1341.

[7] Schiffman JM. Load carriage: Quiet stance, postural sway, and balance recovery. Presentation: Natick Soldier Systems Center, Natick, MA. 2004.

[8] Cavanagh P, Lafortune MA. Ground reaction forces in distance running. Journal of Biomechanics 1980; 13: 397-406.

[9] Knapik J. Discharges during US Army basic training: Injury rates and risk factors. Military Medicine 2001; 166: 641-647.

[10] Jones R, Llewellyn M, Collins S. Using ground reaction forces to guide military physical training. Journal of Defence Science 2001; 6: 219-227. 
[11] Hsiang SM, Chang C. The effect of gait speed and load carrying on the reliability of ground reaction forces. Safety Science 2002; 40: 639-657. 
A) Vertical

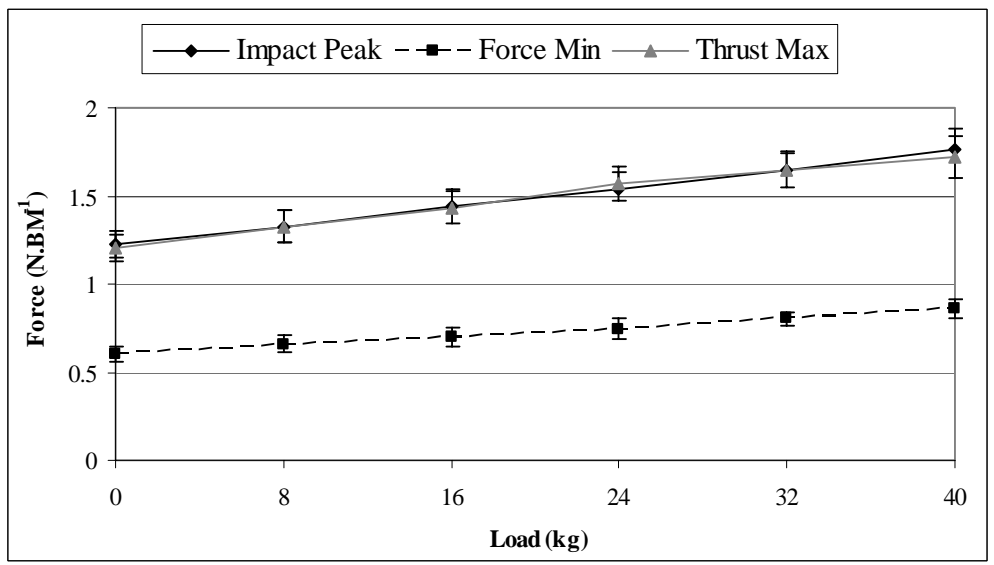

B) Anteroposterior

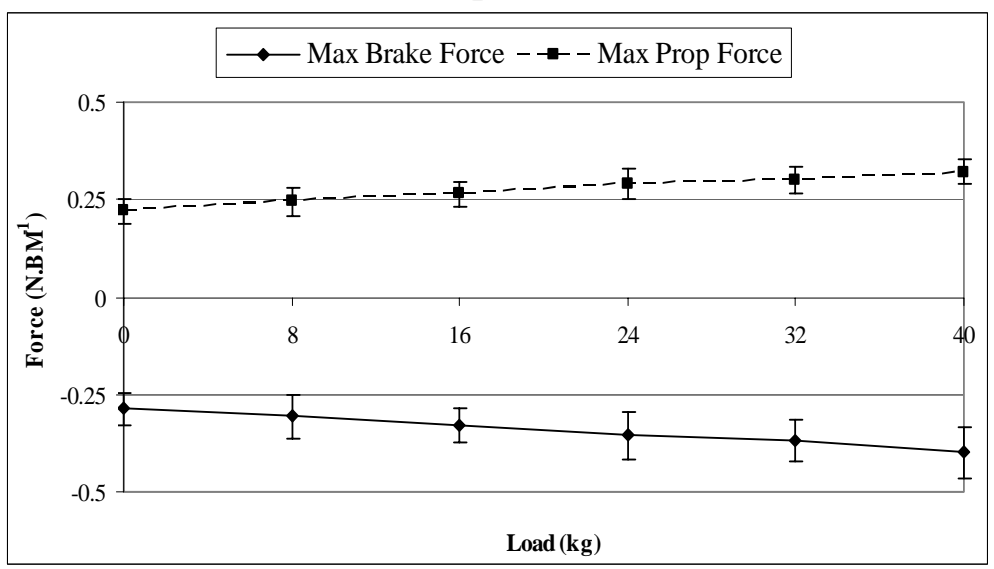

Figure 1: Changes to mean vertical (A) and anteroposterior (B) GRF parameters with increase in load, errors bars are represented by the standard deviation of the data. 
Table 1: Description of the conditions used during the trial and total load carried.

\begin{tabular}{|c|c|c|}
\hline Condition & Description & Load \\
\hline Boot & Wearing non-restrictive clothes and military boots & $0 \mathrm{~kg}$ \\
Rifle & As Boot, but carrying a replica SA80 rifle & $0 \mathrm{~kg}$ \\
Webbing 1 & As Rifle, with the addition of 8kg webbing & $8 \mathrm{~kg}$ \\
Webbing 2 & As Webbing 1, increasing load to 16 kg & $16 \mathrm{~kg}$ \\
Backpack & As Rifle, with the addition of 16 kg Bergen & $16 \mathrm{~kg}$ \\
LCS 1 & As Rifle, carrying 8 kg webbing and 16 kg Bergen & $24 \mathrm{~kg}$ \\
LCS 2 & As Rifle, carrying 16 kg webbing and 16 kg Bergen & $32 \mathrm{~kg}$ \\
LCS 3 & As LCS 2, with addition of 8 kg in the Bergen & $40 \mathrm{~kg}$ \\
\hline
\end{tabular}


Table 2: Results showing changes to mean GRF parameters with the addition of $8 \mathrm{~kg}$ increments of load from 0 to $40 \mathrm{~kg}$, standard deviation in parentheses. Significance derived from the overall effect of load on selected parameter, * indicates significance ( $\mathrm{p} \leq 0.05)$. Forces are measured in $\left(\mathrm{N} . \mathrm{BW} \mathrm{W}^{-1}\right)$, Impulses and Rates in $\left(\left(\mathrm{N} \cdot \mathrm{BW}^{-1}\right) . \mathrm{s}\right)$ and Time in $(\mathrm{s})$.

\begin{tabular}{|c|cccccc|c|}
\hline GRF Parameter & Condition & Level of \\
& Rifle & Webbing 1 & Webbing 2 & LCS 1 & LCS 2 & LCS 3 & Significance \\
\hline Impact Peak & $1.226(0.08)$ & $1.327(0.08)$ & $1.443(0.09)$ & $1.541(0.11)$ & $1.650(0.11)$ & $1.763(0.13)$ & $\mathrm{p} \leq 0.001 *$ \\
Force Minimum & $0.602(0.05)$ & $0.644(0.05)$ & $0.697(0.06)$ & $0.741(0.06)$ & $0.795(0.04)$ & $0.854(0.05)$ & $\mathrm{p} \leq 0.001 *$ \\
Thrust Maximum & $1.205(0.08)$ & $1.326(0.09)$ & $1.434(0.09)$ & $1.571(0.09)$ & $1.645(0.10)$ & $1.721(0.12)$ & $\mathrm{p} \leq 0.001 *$ \\
Max Braking Force & $-0.287(0.04)$ & $-0.306(0.06)$ & $-0.334(0.04)$ & $-0.356(0.06)$ & $-0.368(0.06)$ & $-0.399(0.07)$ & $\mathrm{p} \leq 0.001 *$ \\
Max Propulsive Force & $0.222(0.03)$ & $0.246(0.04)$ & $0.266(0.03)$ & $0.289(0.04)$ & $0.300(0.03)$ & $0.321(0.03)$ & $\mathrm{p} \leq 0.001 *$ \\
Vertical Impulse & $1.076(0.05)$ & $1.191(0.07)$ & $1.288(0.06)$ & $1.411(0.08)$ & $1.492(0.09)$ & $1.595(0.10)$ & $\mathrm{p} \leq 0.001 *$ \\
Mediolateral Impulse & $0.043(0.01)$ & $0.048(0.01)$ & $0.050(0.01)$ & $0.052(0.01)$ & $0.053(0.01)$ & $0.056(0.01)$ & $\mathrm{p}=0.043 *$ \\
Stance Time & $0.663(0.02)$ & $0.674(0.02)$ & $0.676(0.02)$ & $0.689(0.02)$ & $0.689(0.02)$ & $0.692(0.03)$ & $\mathrm{p}=0.003 *$ \\
\hline
\end{tabular}


Table 3: Results showing changes to selected mean GRF parameters for rifle carriage (boot and rifle condition) and load distribution (webbing 2 and backpack condition), standard deviation in parentheses. * indicates significant difference between conditions ( $\mathrm{p} \leq 0.05)$.

\begin{tabular}{|c|cc|c|cc|c|}
\hline GRF Parameter & \multicolumn{2}{|c|}{ Condition } & Level of & Condition & Level of \\
& Boot & Rifle & Significance & Webbing 2 & Backpack & Significance \\
\hline Impact Peak & $1.203(0.09)$ & $1.226(0.08)$ & $\mathrm{p}=0.029 *$ & $1.443(0.09)$ & $1.409(0.10)$ & $\mathrm{p}=0.010 *$ \\
Force Minimum & $0.622(0.06)$ & $0.602(0.05)$ & $\mathrm{p}=0.018 *$ & $0.697(0.06)$ & $0.703(0.05)$ & $\mathrm{p}>0.05$ \\
Thrust Maximum & $1.212(0.09)$ & $1.205(0.08)$ & $\mathrm{p}>0.05$ & $1.434(0.09)$ & $1.443(0.09)$ & $\mathrm{p}>0.05$ \\
Max Braking Force & $-0.286(0.05)$ & $-0.287(0.04)$ & $\mathrm{p}>0.05$ & $-0.334(0.04)$ & $-0.338(0.05)$ & $\mathrm{p}>0.05$ \\
Max Propulsive Force & $0.215(0.03)$ & $0.222(0.03)$ & $\mathrm{p}=0.011 *$ & $0.266(0.03)$ & $0.264(0.04)$ & $\mathrm{p}>0.05$ \\
Vertical Impulse & $1.082(0.06)$ & $1.076(0.05)$ & $\mathrm{p}>0.05$ & $1.288(0.06)$ & $1.297(0.07)$ & $\mathrm{p}>0.05$ \\
Mediolateral Impulse & $0.040(0.01)$ & $0.043(0.01)$ & $\mathrm{p}=0.025 *$ & $0.050(0.01)$ & $0.047(0.01)$ & $\mathrm{p}>0.05$ \\
Stance Time & $0.662(0.02)$ & $0.663(0.02)$ & $\mathrm{p}>0.05$ & $0.676(0.02)$ & $0.687(0.02)$ & $\mathrm{p}=0.002 *$ \\
\hline
\end{tabular}

